\title{
OPEN The Effect of Early Goal-Directed Therapy on Outcome in Adult Severe Sepsis and Septic Shock Patients: A Meta-Analysis of Randomized Clinical Trials
}

\author{
Jing-Yuan Xu, MD, Qi-Hong Chen, MD, Song-Qiao Liu, MD, Chun Pan, MD, Xiu-Ping Xu, MD, Ji-Bin Han, MD, \\ Jian-Feng Xie, MD, Ying-Zi Huang, MD, Feng-Mei Guo, MD, Yi Yang, MD, PhD, and Hai-Bo Qiu, MD, PhD
}

\begin{abstract}
BACKGROUND: Whether early goal-directed therapy (EGDT) improves outcome in severe sepsis and septic shock remains unclear. We performed a meta-analysis of existing clinical trials to examine whether EGDT improved outcome in the resuscitation of adult sepsis patients compared with control care.

METHODS: We searched for eligible studies using MEDLINE, Elsevier, Cochrane Central Register of Controlled Trials, and Web of Science databases. Studies were eligible if they compared the effects of EGDT versus control care on mortality in adult patients with severe sepsis and septic shock. Two reviewers extracted data independently. Data including mortality, sample size of the patients with severe sepsis and septic shock, and resuscitation end points were extracted. Data were analyzed using methods recommended by the Cochrane Collaboration Review Manager 4.2 software. Random errors were evaluated by trial sequential analysis (TSA). RESULTS: Nine studies compared EGDT with control care, and 5202 severe sepsis and septic shock patients were included. A nonsignificant trend toward reduction in the longest all-cause mortality was observed in the EGDT group compared with control care (relative risk, 0.89; 99\% confidence interval, 0.74-1.07; $P=0.10$ ). However, EGDT significantly reduced intensive care unit mortality in severe sepsis and septic shock patients (relative risk, 0.72; 99\% confidence interval, 0.57-0.90; $P=0.0002$ ). TSA indicated lack of firm evidence for a beneficial effect. CONCLUSIONS: In this meta-analysis, a nonsignificant trend toward reduction in the longest all-cause mortality in patients resuscitated with EGDT was noted. However, EGDT significantly reduced intensive care unit mortality in severe sepsis and septic shock patients. TSA indicated a lack of firm evidence for the results. More powered, randomized controlled trials are needed to determine the effects. (Anesth Analg 2016;123:371-81)
\end{abstract}

\begin{abstract}
S evere sepsis and septic shock are major causes of death in critically ill patients, ${ }^{1}$ with a mortality rate of $14 \%$ to $40 \% .^{2}$ Increased compliance with sepsis performance bundles is associated with a reduction in mortality.,4 As fundamental principles for sepsis management, early recognition, control of infection, early and appropriate administration of antibiotics, and resuscitation with IV fluids and vasoactive drugs are widely accepted by intensivists. ${ }^{5}$ Early adequate hemodynamic resuscitation is emphasized, as the key elements should be focused on saving lives.
\end{abstract}

From the Department of Critical Care Medicine, Nanjing Zhongda Hospital School of Medicine, Southeast University, Nanjing, P.R. China.

Accepted for publication February 3, 2016

Funding: This work is partially supported by grants from the National Natural Science Foundations of China (81170057, 81201489, 81300043, 81300060, 81372093, and 81501705), grants from the Clinical Medicine Science and Technology program of Jiangsu Province (BL2013030).

The authors declare no conflicts of interest

Supplemental digital content is available for this article. Direct URL citations appear in the printed text and are provided in the HTML and PDF versions of this article on the journal's website (www.anesthesia-analgesia.org)

Reprints will not be available from the authors.

Address correspondence to Hai-Bo Qiu, MD, PhD, Department of Critical Care Medicine, Nanjing Zhongda Hospital, School of Medicine, Southeast University, 87 Dingjiaqiao Rd, Nanjing 210009, P.R. China. Address e-mail to haiboq2000@163.com.

Copyright $\odot 2016$ International Anesthesia Research Society. This is an openaccess article distributed under the terms of the Creative Commons Attribution-Non Commercial-No Derivatives License 4.0 (CCBY-NC-ND), where it is permissible to download and share the work provided it is properly cited. The work cannot be changed in any way or used commercially.

DOI: 10.1213/ANE.0000000000001278
A proof-of-concept trial ${ }^{6}$ found that, when compared with a control protocol, early hemodynamic resuscitation with a specific protocol termed early goal-directed therapy (EGDT) improved outcomes in patients with severe sepsis. Several additional studies using a similar protocol involving central venous pressure, mean arterial pressure, and central venous oxygen saturation $\left(\mathrm{ScvO}_{2}\right)$ to guide hemodynamic resuscitation also found a survival benefit with EGDT. ${ }^{-9}$ As a result, EGDT principles are subsequently incorporated into the early hemodynamic resuscitation bundle of the Surviving Sepsis Campaign guidelines. ${ }^{5}$ However, in 3 multicenter randomized trials $^{10-12}$ (Protocolized Care for Early Septic Shock [ProCESS] study, the Australasian Resuscitation in Sepsis Evaluation [ARISE] study, and Protocolized Management in Sepsis [ProMISe] trial) published recently, EGDT did not decrease mortality in severe sepsis and septic shock when compared with control care.

The controversial question of whether EGDT improves outcome of severe sepsis and septic shock thus remains relevant. Moreover, whether specific patient characteristics are associated with a potential benefit of EGDT is also unclear. Finally, whether all elements of the protocol are necessary in the hemodynamic resuscitation of severe sepsis and septic shock is unknown. Our goal was to perform a meta-analysis to examine whether EGDT improved outcome when employed in the resuscitation of adult patients with severe sepsis and septic shock compared with control therapy. 


\section{METHODS}

\section{Approval}

Our IRB does not require ethics approval for systematic reviews, including network meta-analyses, because there are no data being collected from patients. We evaluated and synthesized only data in published trials.

\section{Search Strategy for Identification of Relevant Studies}

We searched the following databases: Medline, Elsevier, Cochrane Central Register of Controlled Trials, and Web of Science databases. The following keywords were used as searching terms: "goal-directed" or "goal-directed resuscitation" or "early goal-directed therapy" or "EGDT" or "bundle" or "sepsis bundle" and "sepsis" or "severe sepsis" or "septic shock" or "shock" or "critical ill" or "critical illness" or "intensive care units" or "intensive care" or "critical care" or "ICU." No language restrictions were placed on the search. All databases were searched for articles published from inception until March 17, 2015. Additional files and supplementary appendices of the relevant articles were also reviewed. Detailed search strategies are shown in Supplemental Digital Content 1 (http:/ / links.lww.com/AA/B409).

\section{Study Selection}

One reviewer screened the search results, and the fulltext manuscripts of all potentially eligible studies were acquired. All the articles were then reviewed by 2 reviewers independently in accordance with the inclusion and exclusion criteria. Twelve disagreements between the 2 reviewers were resolved by consensus and discussion including a third reviewer. Any inconsistency in study inclusion and exclusion and their reason lead to the discussion. Details of the consensus are shown in Supplemental Digital Content 2 (http:/ /links.lww.com/ AA/B410).

\section{Inclusion and Exclusion Criteria}

We included trials with the following features:

1. Type of trials: randomized controlled clinical trials.

2. Population: trials including adult population with severe sepsis and septic shock. Severe sepsis was defined as sepsis plus sepsis-induced organ dysfunction or tissue hypoperfusion. ${ }^{5}$

3. Intervention: patients submitted to EGDT, which used the protocol involving central venous pressure, mean arterial pressure, and $\mathrm{ScvO}_{2}$ to guide hemodynamic resuscitation.

4. Comparison: control care, including usual care, protocol-based therapy, etc.

5. Outcome: the primary outcome was all-cause mortality, including 28-day mortality, 90-day mortality, or mortality at other time points.

Trials were excluded because of following reason:

1. If they were not published in English or Chinese.

2. If they were not published as original articles.

3. If they did not enroll adult patients.

4. If they did not compare EGDT with control care.
5. If they included no data on mortality in patients with severe sepsis and septic shock.

6. If full-text articles were not available.

\section{Quality Assessment}

The quality of each article was assessed by 2 reviewers independently. Disagreement that occurred once was resolved by consulting a third reviewer. The 5-point Jadad scale ${ }^{13}$ was calculated to assess the quality of the trial. This scale includes the method of randomization, blinding, and loss to follow-up. In addition, sequences generation, allocation concealment, incomplete outcome data, selective reporting, and other bias were also inspected to assess the risk of bias. The latter was reported as low risk, unclear risk, or high risk for each trial. Low risk was defined as low risk of bias in all domains. Unclear risk was defined as unclear risk of bias in at least 1 domain with no high risk of bias domains. High risk was defined as high risk of bias in 1 or more domains.

\section{Data Extraction and Management}

Using a data extraction table, 2 reviewers independently extracted data. Disagreements that occurred twice were resolved by discussion with another reviewer until a consensus was achieved. Then, data were proofread by another reviewer.

Mortality data were recorded as primary predefined outcome parameters during the data extraction. When $>1$ value for mortality was provided by the article, the mortality for the longest complete follow-up was preferentially used in the meta-analysis. If no 28-day or 90-day mortality values were presented, intensive care unit (ICU) or hospital mortality, or mortality at other time points, was recorded. Ninety-day mortality was reported by the ProCESS study, ${ }_{1}^{10}$ the ARISE study, ${ }_{11}^{11}$ and the ProMISe study. ${ }^{12}$ Sixty-day mortality was reported by the study of Rivers et al. ${ }^{6}$ Twenty-eight-day mortality was reported by the study of Yan et al. ${ }^{14}$ Fourteen-day mortality was reported by the study of Wang et al. ${ }^{15}$ In-hospital mortality was reported by the study of Rivers et al, ${ }_{1}^{6}$ the ProCESS study, ${ }^{10}$ the ARISE study, ${ }^{11}$ the ProMISe study, ${ }^{12}$ the study of Jones et $\mathrm{al}_{1}{ }^{16}$ and the study of Lu et al. ${ }^{17}$ ICU mortality was reported by the ARISE study, ${ }^{11}$ the study of Yan et $\mathrm{al}^{11}{ }^{14}$ the study of Wang et al, ${ }^{15}$ and the study of Chen et al. ${ }^{18}$

Other data, including ICU length of stay, study characteristics, inclusion and exclusion criteria, sample size of the trial, resuscitation end points, and detailed information, were extracted as secondary predefined outcome parameters. If there was insufficient information in the publications, the authors were contacted to obtain missing information.

The effects of EGDT on all-cause mortality, hospital mortality, ICU mortality, and ICU length of stay in severe sepsis and septic shock patients were observed in the meta-analysis. Then effects of EGDT on mortality in severe sepsis and septic shock patients with different severity of illness and with/without venous oxygen saturation were evaluated.

\section{Statistical Analysis}

Data were analyzed by Review Manager 4.2 (The Nordic Cochrane Center, Rigshospitalet, Copenhagen, Denmark). The relative risk for dichotomous data and mean differences for continuous data with $99 \%$ confidence intervals (CIs) were calculated. The statistical heterogeneity of the data 
was explored and quantified using the $I^{2}$ test. Heterogeneity was predefined as $P<0.05$. $\mathrm{I}^{2}$ value of $0 \%$ to $24.9 \%, 25 \%$ to $49.9 \%, 50 \%$ to $74.9 \%$, and $75 \%$ to $100 \%$ were considered as none, low, moderate, and high thresholds, respectively. ${ }^{19,20}$ The randomized-effects model was used if heterogeneity was observed ${ }^{21}$; otherwise, the fixed-effects model was used. To explore the significant heterogeneity, sensitivity analyses were performed. Results were considered statistically significant at 2 -sided $P<0.01$.

Most continuous data were displayed by mean \pm SD. However, the ICU length of stay was reported by median and interquartile ranges in ARISE ${ }^{11}$ and ProMISe trial, ${ }^{12}$ with large sample sizes. As a result, we used median instead of mean, and SD calculated by interquartile range divided by 1.35 .

Predefined subgroup analysis was conducted comparing EGDT protocols with and without $\mathrm{S}_{\mathrm{CVO}}$. In addition, we performed post hoc analyses according to setting (emergency department versus ICU), timing (within 6 hours versus unclear), and fluids (fluid resuscitation volume in 6 hours $>4$ L versus fluid volume in 6 hours $<4 \mathrm{~L}$ ).

With the type I errors resulting from an increased risk of error and repeated significance testing, trial sequential analysis (TSA; TSA software version 0.9 Beta; Copenhagen Trial Unit, Copenhagen, Denmark) was performed to combine information size estimation with an adjusted threshold for statistical significance in the cumulative meta-analysis. Information size was calculated as diversity-adjusted information size, suggested by the relative risk reduction of the intervention in the included studies.

\section{RESULTS}

\section{Study Location and Selection}

A total of 1135 records were identified through the initial search, and 149 were removed as duplicates. The remainder of the 986 records was screened. After assessment of the titles and abstracts, 943 articles were excluded as irrelevant; 2 fulltext articles were unavailable. The flow diagram is presented in Figure 1. In total, 41 potentially eligible studies were identified and 32 of these were excluded, leaving 9 studies $^{6,10-12,14-18}$ that met inclusion criteria and compared EGDT with control

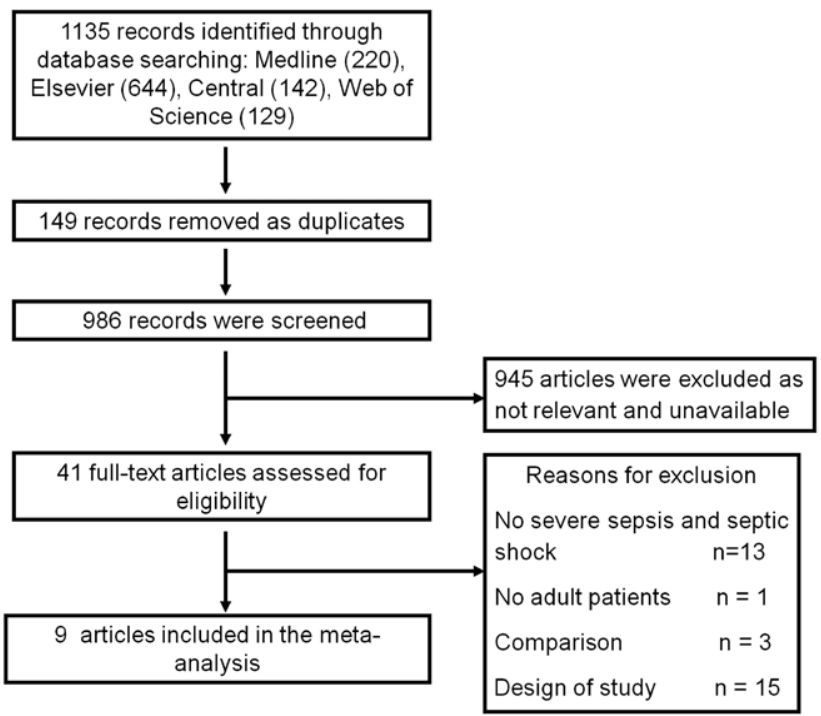

Figure 1. Flow diagram of the search process and study selection. care. Detailed excluded articles are listed in Supplemental Digital Content 2 (http:/ /links.lww.com/AA/B410).

\section{Study Characteristics}

The characteristics of the included studies are shown in Supplemental Digital Content 3 (http://links.lww.com/ AA/B411). The meta-analysis included 5202 severe sepsis and septic shock patients, 2382 in the EGDT group and 2820 in the control group. Among the 9 trials, $5^{10-12,14,16}$ were multiple-center studies, and the remaining $4^{6,15,17,18}$ were singlecenter studies. Four trials ${ }^{14,15,17,18}$ were conducted in the ICU, and the remaining $5^{6,10-12,16}$ were conducted in the emergency department. Seven trials ${ }^{6,10-12,14,17,18}$ enrolled and resuscitated patients within 6 hours, and 2 trials ${ }^{15,16}$ reported unclear timing of enrollment. The severity of disease was reported in each study by the acute physiology and chronic health evaluation II score (APACHE II score), and data were presented as mean \pm SD or medians (interquartile ranges). The distinction between higher severity of disease and less severity was differentiated by us according to the means of APACHE II scores in each article. The APACHE II scores of the included patients in 5 trials $6,10,14,15,17$ were $>20 ; 3^{11,12,18}$ of the remaining were $<20$ or unclear. The fluid volume for resuscitation of the included patients in 2 trials $^{6,15}$ was $>4 \mathrm{~L}$, in 5 trials ${ }^{10-12,16,17}$ was $<4 \mathrm{~L}$, and in the other 2 trials ${ }^{14,18}$ was unclear.

Details of intervention used in included studies are shown in Table 1. Intervention goals of the EGDT group included CVP $\geq 8$ to $12 \mathrm{~mm} \mathrm{Hg}, \mathrm{MAP} \geq 65 \mathrm{~mm} \mathrm{Hg}$, and $\mathrm{ScvO}_{2}$ $\geq 70 \%$ in all included studies. Four trials $6,14,16,18$ used the protocol, including identical goal of CVP and MAP but without $\mathrm{SCVO}_{2}$ as control care, the other 5 trials ${ }^{10-12,15,17}$ used control care with different protocols.

Quality assessment of the included studies is shown in Table 2. Three trials ${ }^{6,10,16}$ were judged to be at low risk of bias or unclear risk of bias, and the remaining 6 trials $^{11,12,14,15,17,18}$ were judged to be at high risk of bias. None of the 9 studies was double-blinded because of the extreme difficulty in blinding required to evaluate the complex intervention such as EGDT. However, we judged that mortality and ICU length of stay were not likely to be influenced by lack of blinding.

\section{The Effects of EGDT on Mortality in Severe Sepsis and Septic Shock Patients}

The effects of EGDT on mortality in patients with severe sepsis were estimated from 9 trials (Figure 2), and the heterogeneity was observed $\left(P=0.04, I^{2}=51.6 \%\right)$. The longest complete follow-up mortality rates of all the trials were evaluated in the analysis of all-cause mortality. The overall mortality in the EGDT and control group was 636 of 2382 (26.7\%) and 831 of 2820 (29.5\%), respectively. No significantly reduced all-cause mortality was observed in EGDT group compared with control care (relative risk [RR], 0.89; 99\% CI, 0.74-1.07; P = 0.10).

\section{The Effects of EGDT on Hospital Mortality in Severe Sepsis and Septic Shock Patients} Six trials ${ }^{6,10-12,16,17}$ reported hospital mortality in patients with severe sepsis and septic shock. Hospital mortality was not significantly different between EGDT and control care (RR, 0.98; 99\% CI, 0.78-1.24; $P=0.86$ ). 

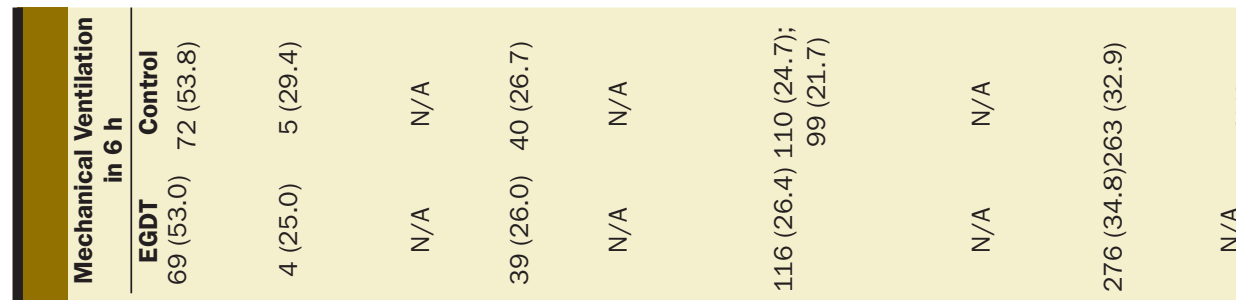

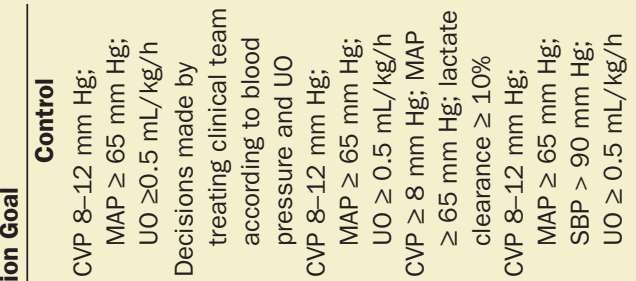
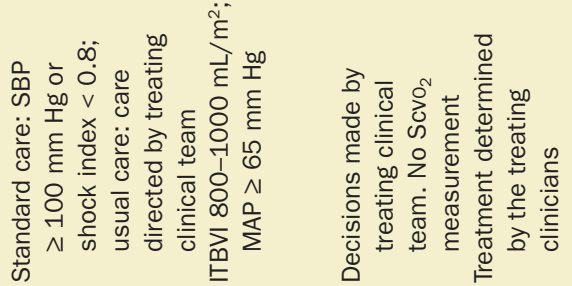

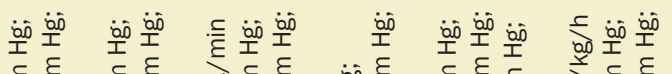

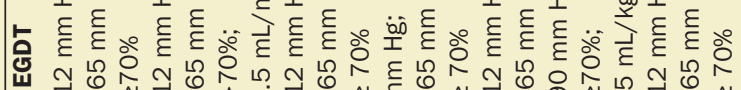

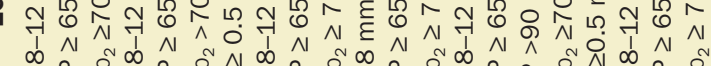

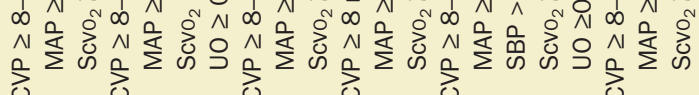

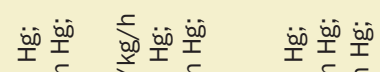

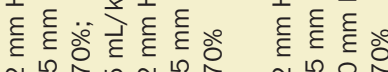

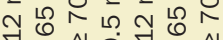

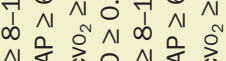

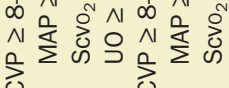

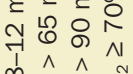

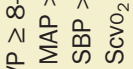

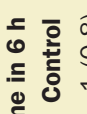

$\stackrel{\bar{m}}{\stackrel{n}{n}} \ll$

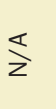

नें

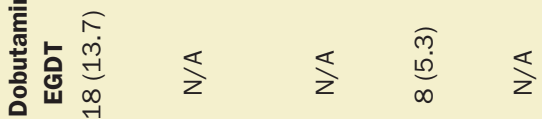

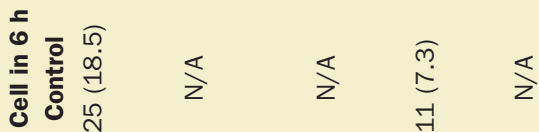

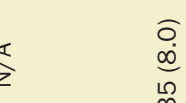

$\underset{\substack{0 \\ \infty}}{\infty}$

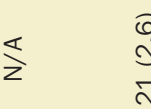

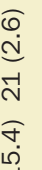

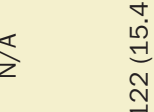



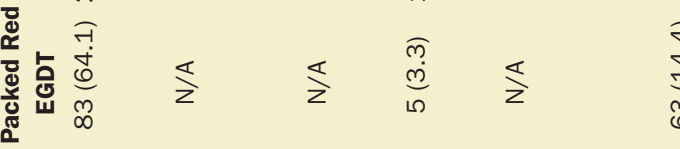

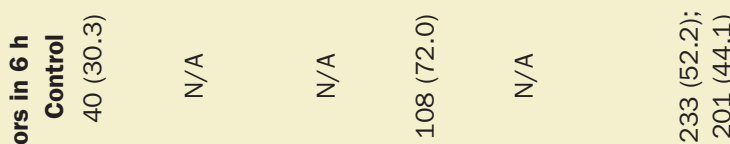

กิ-

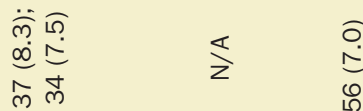

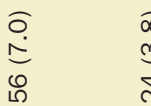

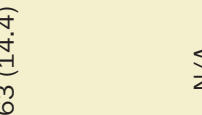

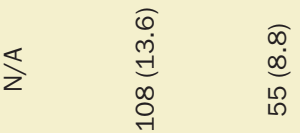

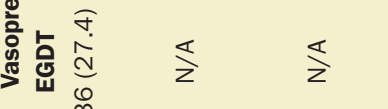

$\stackrel{\substack{m \\ \stackrel{m}{\infty}}}{\substack{n \\ 0}}$

$\frac{2}{2}$

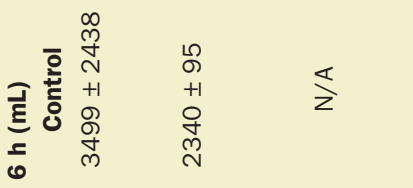

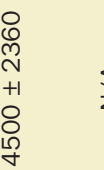

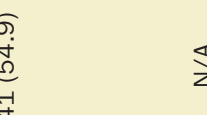

$\stackrel{1}{\Sigma}$

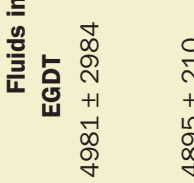

$\ll$

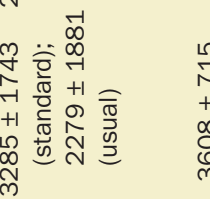

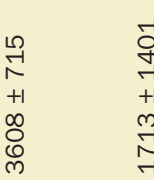

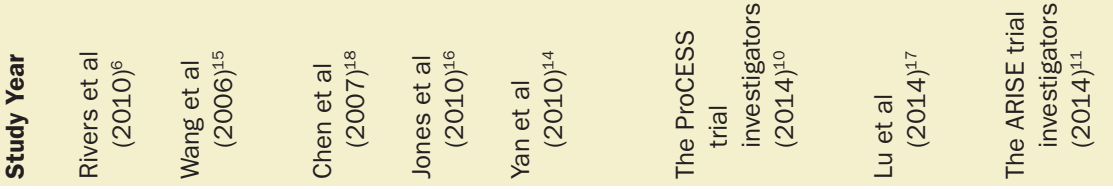

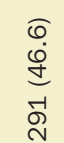

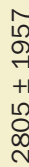

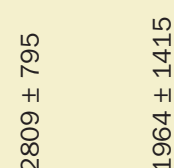

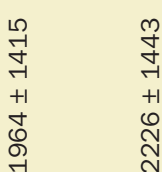

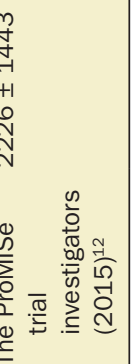

要

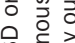

$+\frac{1}{4}+\frac{1}{4}$ 


\begin{tabular}{|c|c|c|c|c|c|c|c|c|}
\hline Authors & $\begin{array}{l}\text { Sequences } \\
\text { Generation }\end{array}$ & $\begin{array}{l}\text { Allocation } \\
\text { Concealment }\end{array}$ & $\begin{array}{l}\text { Blinding of } \\
\text { Participants and } \\
\text { Researchers }\end{array}$ & $\begin{array}{l}\text { Blinding of } \\
\text { Outcome } \\
\text { Assessment }\end{array}$ & $\begin{array}{l}\text { Incomplete } \\
\text { Outcome } \\
\text { Data }\end{array}$ & $\begin{array}{l}\text { Selective } \\
\text { Reporting }\end{array}$ & $\begin{array}{l}\text { Other } \\
\text { Bias }\end{array}$ & $\begin{array}{l}\text { Overall Risk } \\
\text { of Bias }\end{array}$ \\
\hline Rivers et al $(2001)^{6}$ & Low & Low & Low & Low & Low & Low & Low & Low \\
\hline Wang et al $(2006)^{15}$ & Unclear & Unclear & High & High & Low & Unclear & Low & High \\
\hline Chen et al $(2007)^{18}$ & Unclear & Unclear & High & High & Unclear & Unclear & Low & High \\
\hline Jones et al $(2010)^{16}$ & Low & Low & Low & Low & Low & Unclear & Low & Unclear \\
\hline Yan et al $(2010)^{14}$ & Low & Unclear & High & High & Low & Unclear & Low & High \\
\hline $\begin{array}{l}\text { The ProCESS study } \\
\text { investigators }(2014)^{10}\end{array}$ & Low & Low & Low & Low & Low & Low & Low & Low \\
\hline Lu et al $(2014)^{17}$ & Low & Unclear & High & High & Low & Unclear & Low & High \\
\hline $\begin{array}{l}\text { The ARISE study investigators } \\
\qquad(2014)^{11}\end{array}$ & Low & Low & High & High & Low & Low & Low & High \\
\hline $\begin{array}{l}\text { The ProMISe study } \\
\text { investigators }(2015)^{12}\end{array}$ & Low & Low & High & High & Low & Low & Low & High \\
\hline
\end{tabular}

Sequence generation: Rivers et $a^{6}$ used computer-generated blocks of 2 to 8 for randomization. Random numbers was used by the study of Yan et al ${ }^{14}$ and of Lu et al. ${ }^{17}$ No detailed sequence generation was reported by the study of Wang et al ${ }^{15}$ and Chen et al. ${ }^{18}$ The group assignment sequence was generated by an independent statistician using a parallel design, balanced randomization schedule in the study of Jones et al. ${ }^{16}$ Randomization was performed with the use of a centralized Web-based program in variable block sizes of 3, 6 , or 9 by the ProCESS study. ${ }^{10}$ Randomization was stratified according to study center with the use of a permuted block method and was performed by means of a centralized telephone interactive voice-response system that was accessible 24 hours a day by the ARISE study. ${ }^{11}$ Study-group assignment was performed by means of randomized permuted blocks, with variable block lengths of 4,6 , and 8 , and stratified according to site by the ProMISe study. ${ }^{12}$

Allocation concealment: No detailed allocation concealment was reported by Wang et al, ${ }^{15}$ Chen et al, ${ }^{18}$ Yan et al, ${ }^{14}$ and Lu et al. ${ }^{17}$ Rivers et al ${ }^{6}$ and Jones et al ${ }^{16}$ used sealed, opaque, randomly assorted envelopes. Stratification according to site and race with the use of a centralized Web-based program was used by the ProCESS study. ${ }^{10}$ Randomization was stratified according to study center with the use of a permuted block method and was performed by means of a centralized telephone interactive voice-response system that was accessible 24 hours a day by the ARISE study. ${ }^{11}$ Study-group assignment was performed by means of randomized permuted blocks, with variable block lengths of 4,6 , and 8 , and stratified according to site by the ProMISe study. ${ }^{12}$

Blinding: Clinicians who subsequently assumed the care of the patients were blinded to the treatment assignment in the study of Rivers et al. ${ }^{6}$ Blinding was not reported by the study of Wang et al, ${ }^{15}$ Chen et al, ${ }^{18}$ Jones et al, ${ }^{16}$ Yan et al, ${ }^{14}$ and Lu et al. ${ }^{17}$ The clinical coordinating team and investigators at the participating sites remained unaware of the study-group outcomes until the data were locked by the ProCESS study. ${ }^{10}$ No blinding to study-group assignment was reported by the ARISE study ${ }^{11}$ and the ProMISe study. ${ }^{12}$

Incomplete outcome data: All trials described the follow-up.

Selective reporting: The study protocols of the ProCESS study, ${ }^{10}$ the ARISE study, ${ }^{11}$ and the ProMISe study ${ }^{12}$ were obtained.

Other bias: No evidence of other sources of bias.

\section{The Effects of EGDT on ICU Mortality in Severe Sepsis and Septic Shock Patients}

Four trials ${ }^{11,14,15,18}$ reported ICU mortality in patients with severe sepsis and septic shock. EGDT significantly reduced ICU mortality in severe sepsis and septic shock patients (RR, 0.72; 99\% CI, 0.57-0.90; $P=0.0002$ ).

\section{The Effects of EGDT on Mortality in Severe Sepsis and Septic Shock Patients With Different Severity of Illness}

For patients with a higher severity of disease (APACHE II score $\geq 20$ ), mortality benefit trended toward EGDT (RR, $0.87 ; 99 \%$ CI, 0.74-1.03; $P=0.03$ ) when compared with control care (Figure 3).

\section{The Effects of Goal-Directed Therapy on Mortality in Severe Sepsis and Septic Shock Patients With and Without Central Venous Oxygen Saturation}

Predefined subgroup analysis according to protocol with versus without $\mathrm{ScvO}_{2}$ suggested no significant difference of mortality between the 2 protocols; however, mortality benefit trended toward $\mathrm{ScvO}_{2}$ monitoring (RR, 0.88; 99\% CI, $0.73-1.06 ; P=0.07$ ) when compared with protocol including identical remaining intervention goals (Figure 4).

\section{Post Hoc Subgroup Analyses for Overall Mortality}

Post hoc subgroup analyses (Table 3) according to the setting of EGDT for resuscitation suggested that mortality benefit was only seen in the subgroup when EGDT was conducted in the ICU (RR, 0.67; 99\% CI, 0.51-0.88; $P=0.0002$ ) but not in the subgroup conducted in the emergency department. Moreover, although there was a lack of statistical significance, more trending toward reduced mortality was found when EGDT was performed within 6 hours than when timing of EGDT was unclear (RR, 0.87; 99\% CI, 0.721.05; $P=0.05)$ or when there was EGDT with more fluid volume in patients with severe sepsis and septic shock (RR, $0.77 ; 99 \%$ CI, 0.55-1.06; $P=0.04$ ). Sensitivity analyses for the effects of EGDT on mortality in severe sepsis and septic shock patients with higher severity of illness and timing were performed (Table 4).

\section{The Effects of EGDT on ICU Length of Stay in Severe Sepsis and Septic Shock Patients}

The ICU length of stay was also evaluated (Figure 5). Five studies $^{10,14-17}$ reported ICU length of stay by mean \pm SD. However, the ICU length of stay was reported by median and interquartile ranges in the ARISE ${ }^{11}$ and the ProMISe trial $^{12}$ with a large sample size. As a result, we used median instead of mean, and SD was calculated by interquartile range divided by 1.35. Compared with control care, EGDT displayed no beneficial effect on ICU length of stay in severe sepsis and septic shock patients.

\section{Random Errors}

To correct for random error and repetitive testing of sparse data, TSA was calculated with $\alpha=0.05$ and $\beta=0.20$ (power $80 \%$ ). The required diversity-adjusted information 


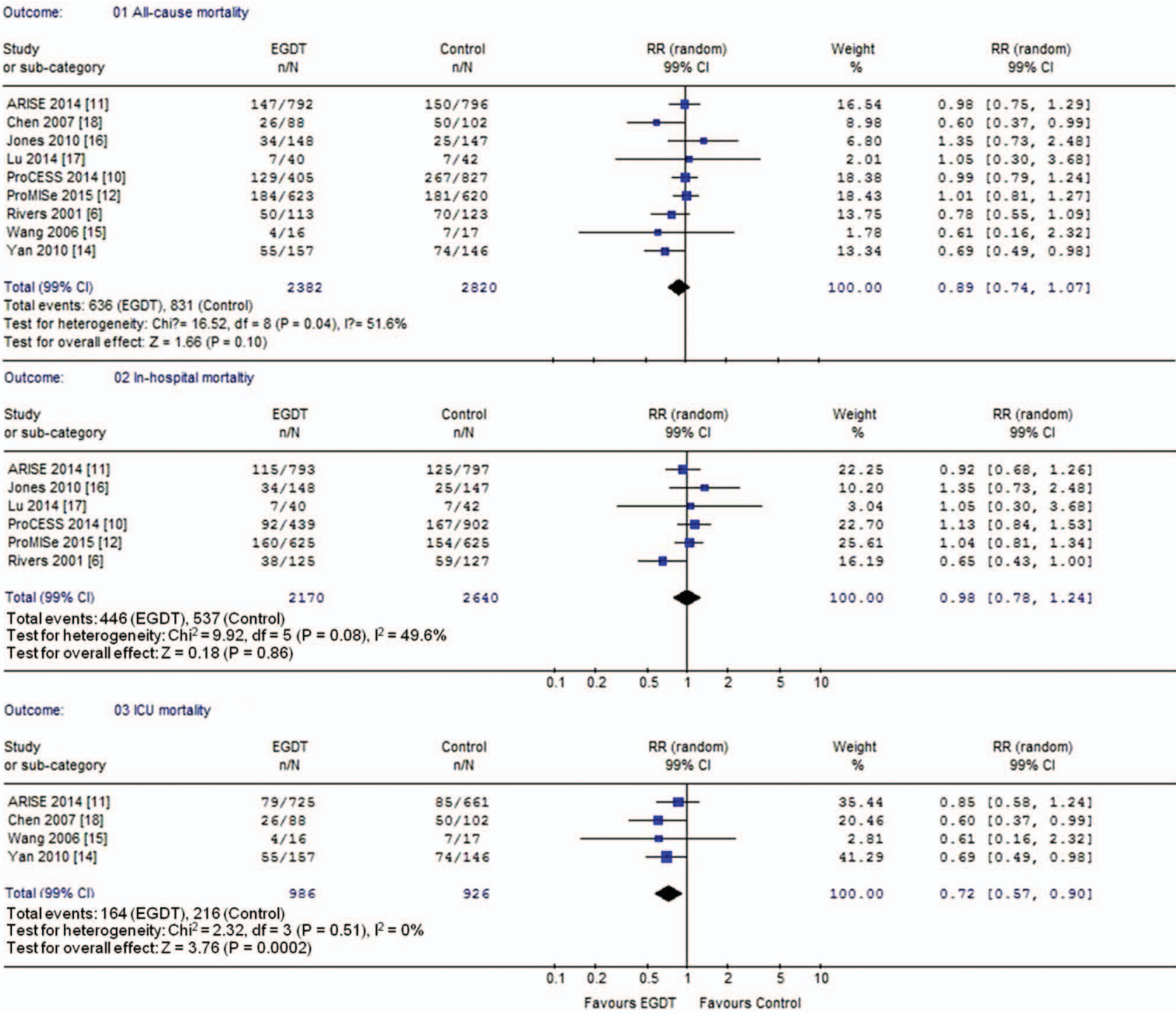

Figure 2. The effects of early goal-directed therapy (EGDT) on mortality in patients with severe sepsis and septic shock. Mortality data were recorded during the data extraction. When there is $>1$ value about mortality published in the article, the longest complete followup was preferentially used for evaluation of all-cause mortality. However, when 28-day or 90-day mortality values were not presented, intensive care unit (ICU) or hospital mortality or mortality at other time points were recorded. Ninety-day mortality was reported by the ARISE study, ${ }^{11}$ the ProCESS study, ${ }^{10}$ and the ProMISe study. ${ }^{12}$ Sixty-day mortality was reported by the study of Rivers et al. ${ }^{6}$ Twenty-eightday mortality was reported by the Yan study. ${ }^{14}$ Fourteen-day mortality was reported by the study of Wang et al. ${ }^{15}$ In-hospital mortality was reported by the study of Rivers et al, ${ }^{6}$ the study of Jones et al, ${ }^{16}$ Lu study, ${ }^{17}$ ARISE study, ${ }^{11}$ ProCESS study, ${ }^{10}$ and ProMISe study. ${ }^{12}$ ICU mortality was reported by the study of Chen et al, ${ }^{18}$ the study of Wang, ${ }^{15}$ Yan study, ${ }^{14}$ and ARISE study. ${ }^{12} \mathrm{CI}$ indicates confidence interval; $\mathrm{RR}$, relative risk.

size based on the intervention effect was suggested by the included trials using a random-effects model (with the relative risk reduction of $9.5 \%$ regarding mortality and 21,342 patients). TSA indicated lack of reliable and conclusive evidence for a beneficial effect of EGDT for the longest complete follow-up mortality in severe sepsis and septic shock patients (Figure 6) because the monitoring boundary was not surpassed and the required information size was not reached.

\section{DISCUSSION}

Our meta-analysis showed no significantly reduced allcause mortality in patients resuscitated with EGDT when compared with control therapy. This meta-analysis differs considerably from those of recently published meta-analyses, but we obtained similar results..$^{22-25}$

In our meta-analysis, TSA indicated lack of firm evidence for our results because of considerable heterogeneity between groups. Our results suggest that although 3 recent multicenter randomized controlled studies reported negative results, conclusive evidence regarding the benefit of EGDT is not possible, and more randomized controlled trials are needed.

Our results, however, suggest that some patient subgroups may benefit from EGDT. In our meta-analysis, for example, we found a trend toward the longest complete 


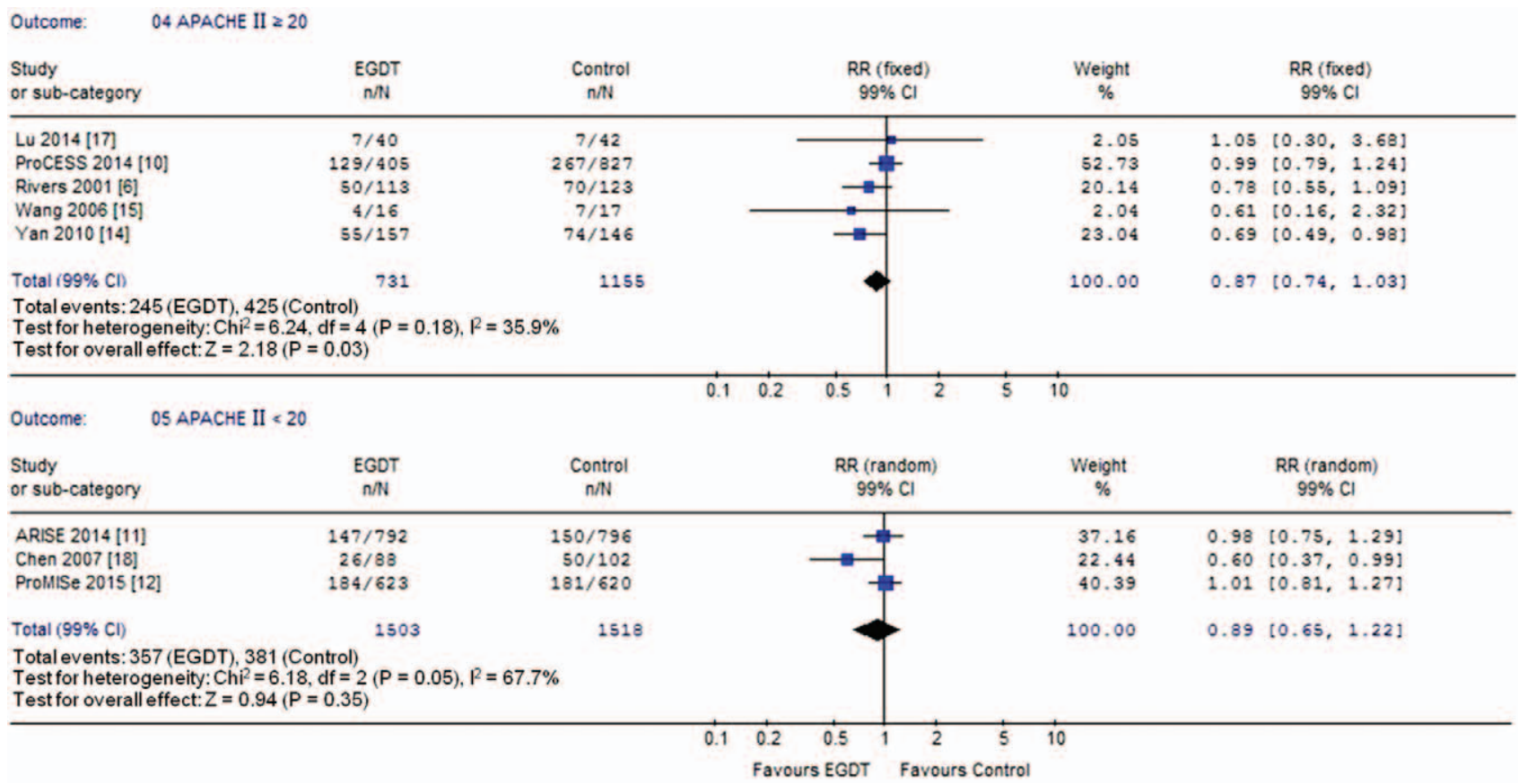

Figure 3. The effects of early goal-directed therapy (EGDT) on mortality in severe sepsis and septic shock patients with different severity of illness. The severity of illness was reported in each study by the acute physiology and chronic health evaluation II score (APACHE II score), and the data were presented as mean \pm SD or medians (interquartile ranges). The distinction between higher severity of illness and less severity was differentiated by us according to the means of APACHE II scores in each article. The APACHE II score of the included patients in 5 trials ${ }^{6,10,14,15,17}$ were $>20$, the remaining $3^{11,12,18}$ were $<20$ or unclear. $\mathrm{Cl}$ indicates confidence interval; RR, relative risk.

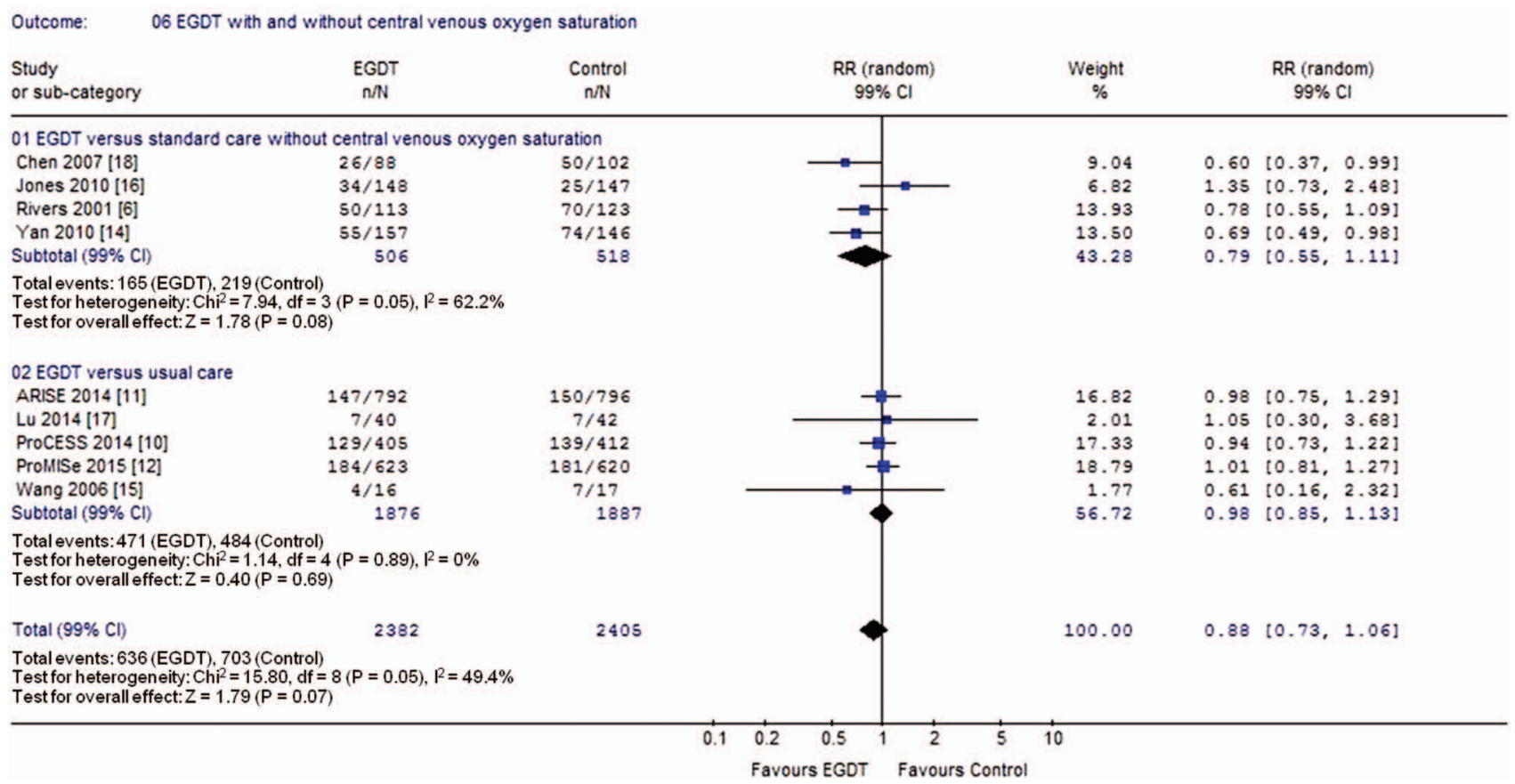

Figure 4. The effects of goal-directed therapy on mortality in severe sepsis and septic shock patients with and without central venous oxygen saturation. Mortality data were recorded during the data extraction. When there is $>1$ value about mortality published in the article, the longest complete follow-up was preferentially used for evaluation of all-cause mortality. However, when 28-day or 90-day mortality values were not presented, intensive care unit (ICU) or hospital mortality or mortality at other time points were recorded. Ninety-day mortality was reported by the ARISE study, ${ }^{11}$ the ProCESS study, ${ }^{10}$ and the ProMISe study. ${ }^{12}$ Sixty-day mortality was reported by the study of Rivers et al. ${ }^{6}$ Twenty-eightday mortality was reported by Yan study. ${ }^{14}$ Fourteen-day mortality was reported by the study of Wang et al. ${ }^{15}$ In-hospital mortality was reported by the study of Rivers et al, ${ }^{6}$ the study of Jones et al, ${ }^{16}$ Lu study, ${ }^{17}$ ARISE study, ${ }^{11}$ ProCESS study,,${ }^{10}$ and ProMISe study. ${ }^{12}$ ICU mortality was reported by the study of Chen et al, ${ }^{18}$ study of Wang et al, ${ }^{15}$ Yan study, ${ }^{14}$ and ARISE study. ${ }^{12} \mathrm{Cl}$ indicates confidence interval; RR, relative risk. 


\begin{tabular}{|c|c|c|c|c|c|}
\hline Subgroups & No. of Studies & No. of Patients & RR (99\% Confidence Interval) & $P$ Value & $I^{2}(\%)$ \\
\hline \multicolumn{6}{|l|}{ Setting } \\
\hline Intensive care unit ${ }^{14,15,17,18}$ & 4 & 608 & $0.67(0.51-0.88)$ & 0.0002 & 0 \\
\hline Emergency department ${ }^{6,10-12,16}$ & 5 & 4594 & $0.97(0.84-1.13)$ & 0.64 & 21.8 \\
\hline \multicolumn{6}{|l|}{ Timing } \\
\hline Within $6 \mathrm{~h}^{6,10-12,14,17,18}$ & 7 & 4874 & $0.87(0.72-1.05)$ & 0.05 & 54.1 \\
\hline Unclear ${ }^{15,16}$ & 2 & 328 & $1.04(0.39-2.73)$ & 0.92 & 48.9 \\
\hline \multicolumn{6}{|l|}{ Fluid volume } \\
\hline More 6,15 & 2 & 269 & $0.77(0.55-1.06)$ & 0.04 & 0 \\
\hline Less $^{10-12,16,17}$ & 5 & 4440 & $1.01(0.88-1.16)$ & 0.84 & 0 \\
\hline
\end{tabular}

Abbreviations: RR, relative risk.

\begin{tabular}{|c|c|c|c|c|c|}
\hline Study Attributes & No. of Studies & No. of Patients & RR (95\% Confidence Interval) & $P$ Value & $I^{2}(\%)$ \\
\hline \multicolumn{6}{|c|}{ Sensitivity analyses for the effects of EGDT on mortality in severe sepsis and septic shock patients with higher severity of illness } \\
\hline $\begin{array}{l}\text { APACHE II > } \\
20^{6,10,14,15,17}\end{array}$ & 5 & 1886 & $0.87(0.74-1.03)$ & 0.03 & 35.9 \\
\hline Rivers et $\mathrm{al}^{6}$ & 4 & 1650 & $0.84(0.60-1.18)$ & 0.19 & 45.3 \\
\hline ProCESS ${ }^{10}$ & 4 & 654 & $0.74(0.58-0.94)$ & 0.001 & 0 \\
\hline Yan et al ${ }^{14}$ & 4 & 1583 & $0.91(0.76-1.10)$ & 0.20 & 0 \\
\hline Wang et $\mathrm{a}^{15}$ & 4 & 1853 & 0.84 (0.64-1.09) & 0.08 & 48.3 \\
\hline Lu et $\mathrm{al}^{17}$ & 4 & 1804 & $0.82(0.62-1.07)$ & 0.05 & 50.5 \\
\hline \multicolumn{6}{|c|}{ Sensitivity analyses for timing of EGDT on mortality in severe sepsis and septic shock patients } \\
\hline Within $6 \mathrm{~h}^{6,10-12,14,17,18}$ & 7 & 4874 & $0.87(0.72-1.05)$ & 0.05 & 54.1 \\
\hline Rivers et $a^{6}$ & 6 & 4638 & $0.88(0.72-1.09)$ & 0.13 & 56.7 \\
\hline ProCESS ${ }^{10}$ & 6 & 3642 & $0.84(0.66-1.05)$ & 0.04 & 57.6 \\
\hline ARISE ${ }^{11}$ & 6 & 3286 & $0.84(0.67-1.05)$ & 0.05 & 59.1 \\
\hline ProMISe $^{12}$ & 6 & 3631 & $0.83(0.67-1.04)$ & 0.03 & 54.0 \\
\hline Yan et $\mathrm{al}^{14}$ & 6 & 4571 & $0.91(0.76-1.09)$ & 0.17 & 42.2 \\
\hline Lu et $\mathrm{al}^{17}$ & 6 & 4792 & $0.86(0.71-1.05)$ & 0.05 & 61.5 \\
\hline Chen et $a^{18}$ & 6 & 4684 & $0.91(0.77-1.07)$ & 0.13 & 40.6 \\
\hline
\end{tabular}

Abbreviations: RR, relative risk.

\begin{tabular}{|c|c|c|c|c|c|c|c|c|}
\hline \multirow{2}{*}{$\begin{array}{l}\text { Outcome: } \\
\text { Study } \\
\text { or sub-category }\end{array}$} & \multicolumn{8}{|c|}{07 ICU length of stay } \\
\hline & N & $\begin{array}{l}\text { EGDT } \\
\text { Mean (SD) }\end{array}$ & N & $\begin{array}{l}\text { Control } \\
\text { Mean (SD) }\end{array}$ & $\begin{array}{l}\text { SMD (random) } \\
\quad 99 \% \mathrm{Cl}\end{array}$ & $\begin{array}{l}\text { Weight } \\
\%\end{array}$ & & $\begin{array}{l}\text { SMD (random) } \\
99 \% \mathrm{Cl}\end{array}$ \\
\hline ARISE 2014 [11] & 793 & $2.80(2.70)$ & 798 & $2.80(3.10)$ & $\frac{1}{1}$ & 20.02 & 0.00 & {$[-0.13,0.13]$} \\
\hline Jones 2010 [16] & 150 & $5.60(7.40)$ & 150 & $5.90(8.50)$ & $\frac{1}{1}$ & 14.95 & -0.04 & {$[-0.34,0.26]$} \\
\hline Lu 2014 [17] & 40 & $9.50(2.50)$ & 42 & $7.10(3.10)$ & $\rightarrow$ & 7.73 & 0.84 & {$[0.25,1.44]$} \\
\hline ProCESS 2014 [10] & 439 & $5.10(6.30)$ & 446 & $5.10(7.10)$ & 1 & 18.83 & 0.00 & {$[-0.17,0.17]$} \\
\hline ProMISe 2015 [12] & 625 & $2.60(3.56)$ & 626 & $2.20(3.93)$ & 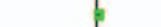 & 19.59 & 0.11 & {$[-0.04,0.25]$} \\
\hline Wang 2006 [15] & 16 & $9.11(2.96)$ & 17 & $11.74(3.39)$ & $\Rightarrow$ & 3.95 & -0.80 & {$[-1.74,0.13]$} \\
\hline $\operatorname{Yan} 2010[14]$ & 157 & $19.90(2.20)$ & 146 & $20.60(1.90)$ & $\Rightarrow$ & 14.93 & -0.34 & {$[-0.64,-0.04]$} \\
\hline Total $(99 \% \mathrm{Cl})$ & 2220 & & 2225 & & $\phi$ & 100.00 & 0.00 & {$[-0.21,0.20]$} \\
\hline \multicolumn{9}{|c|}{$\begin{array}{l}\text { Test for heterogeneity: } \mathrm{Chi}^{2}=30.19, \mathrm{df}=6(\mathrm{P}<0.0001), 1^{2}=80.1 \% \\
\text { Test for overall effect: } Z=0.03(P=0.98)\end{array}$} \\
\hline
\end{tabular}

Figure 5. The effects of early goal-directed therapy (EGDT) on intensive care unit (ICU) length of stay in severe sepsis and septic shock patients. $\mathrm{Cl}$ indicates confidence interval.

follow-up mortality benefit with EGDT in patients with a higher severity of disease (APACHE II score $\geq 20$ ). In addition, EGDT was associated with decreased ICU mortality when compared with control care. These results suggest that EGDT may have some benefit in more critically ill patients.

As the cornerstone for resuscitation in patients with severe sepsis and septic shock, EGDT has been recommended by the Surviving Sepsis Guidelines, ${ }^{5}$ and studies have suggested ${ }^{3}$ that every $10 \%$ increase in compliance is associated with a significant decrease in the odds ratio for mortality. However, the role of EGDT in the treatment of sepsis remains controversial.

Different study time periods may influence the value of EGDT. Earlier trials supported EGDT for severe sepsis and septic shock patients; however, after 15 years, the most recent 3 trials all failed to show any benefit of EGDT. When comparing these studies with the original study of Rivers et al, there were concerns, including the amount of fluids before randomization and the lower overall mortality rate. These concerns could be explained by the broad implementation of, and compliance with, the Guidelines, suggesting 


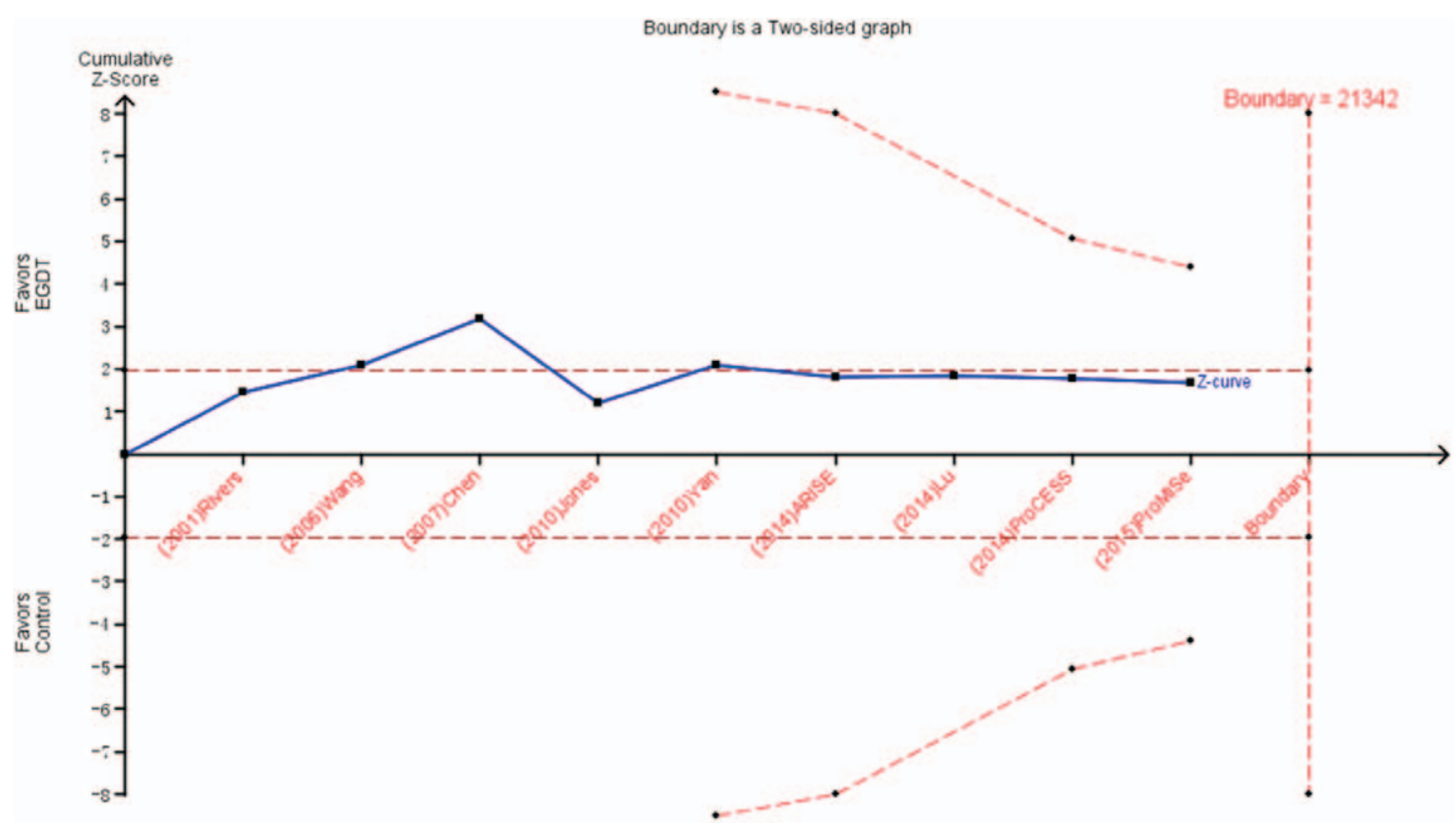

Figure 6. Trial sequential analysis for a relative risk reduction of all-cause mortality of $9.5 \%$ in control group in 9 trials. A required diversityadjusted information size of 21,342 patients was calculated based on a control event proportion of 9.5\%, early goal-directed therapy (EGDT) induced relative risk reduction of mortality of $9.5 \%$ suggested by all trials, $\alpha=0.05$ two-sided, $\beta=0.20$ (power $=80 \%$ ). The cumulated Z-curve (blue) crosses the traditional boundary $(P=0.05)$ but not the trial sequential monitoring boundary, indicating lack of firm evidence for a beneficial effect of EGDT.

that patients with severe sepsis and septic shock need early attention and resuscitation. The earlier we treat sepsis, the better outcome is shown.

Three recent large multicenter trials ${ }^{10-12}$ with lower mortality (ProCESS: $21.0 \%$ vs $18.9 \%$ vs $18.2 \%$; ARISE: $18.6 \%$ vs $18.8 \%$; ProMISe: $29.5 \%$ vs $29.2 \%$ ) compared with the study of Rivers et al (30.5\% vs $46.5 \%$ ) have found no difference in mortality between EGDT and control care, leading to lively discussions. ${ }^{26-29}$ Possible explanations for the difference between trial results include the study population, intervention methods, and goals. The timing and volume of fluids, for example, may play an important role. ${ }^{30}$ As a result, the focus during the golden time of resuscitation for septic shock should be fluid administration. However, in the recent trials, the volume of fluid administration is less (ProCESS: $2805 \pm 1957$ vs $3285 \pm 1743$ vs $2279 \pm 1881 \mathrm{~mL}$; ARISE: $1964 \pm 1415$ vs $1713 \pm 1401 \mathrm{~mL}$; ProMISe: $2226 \pm 1443$ vs $2022 \pm 1271 \mathrm{~mL}$ ) than that in the study of Rivers et al $(4981 \pm 2984$ vs $3499 \pm 2438 \mathrm{~mL})$.

Many EGDT protocols specify using CVP and $\mathrm{ScvO}_{2}$ monitoring to guide management of fluids, vasopressors, packed red-blood-cell transfusions, and dobutamine. Some negative studies ${ }^{16,31}$ have challenged the role of $\mathrm{ScvO}_{2}$ in EGDT protocols and have used lactate clearance instead. Another multicenter study ${ }^{32}$ reported that abnormal $\mathrm{ScvO}_{2}$ values (90\%-100\%) observed in the emergency department were associated with increased mortality, indicating that an $\mathrm{S}_{\mathrm{CVO}}$ target should be achieved in the resuscitation of sepsis.

Our analysis has several limitations. First, only 9 trials were included in our meta-analysis, and some were at high risk of bias. Second, the protocols of control care were different, which may have affected results. Third, different end points were used for mortality evaluation, which may influence the overall results; and ICU length of stay might not be a reliable marker of success because criteria for discharge from the ICU are not uniform.

\section{CONCLUSIONS}

The results of this meta-analysis suggested a nonsignificant trend toward reduction in the longest all-cause mortality in patients resuscitated with EGDT. However, TSA indicated lack of firm evidence for the results. High powered, randomized controlled trials are needed to determine the effects.

\section{DISCLOSURES}

Name: Jing-Yuan Xu, MD.

Contribution: This author performed the analysis and interpretation of data and participated in drafting, editing, and submitting the manuscript.

Name: Qi-Hong Chen, MD.

Contribution: This author reviewed all the articles independently in accordance with the inclusion criteria.

Name: Song-Qiao Liu, MD.

Contribution: This author reviewed all the articles independently in accordance with the inclusion criteria.

Name: Chun Pan, MD.

Contribution: This author was responsible for identifying the clinical question and performing the literature search.

Name: Xiu-Ping Xu, MD.

Contribution: This author was responsible for identifying the clinical question and performing the literature search.

Name: Ji-Bin Han, MD.

Contribution: This author was responsible for extracting methodological data and identifying the approach for data extraction from graphical sources. 
Name: Jian-Feng Xie, MD.

Contribution: This author was responsible for extracting methodological data and identifying the approach for data extraction from graphical sources.

Name: Ying-Zi Huang, MD.

Contribution: This author was responsible for extracting numerical data, performing the data analysis, and interpreting the results. Name: Feng-Mei Guo, MD.

Contribution: This author contributed to study analysis and interpretation of data.

Name: Yi Yang, MD, PhD.

Contribution: This author was responsible for revising the manuscript for important intellectual content.

Name: Hai-Bo Qiu, MD, PhD.

Contribution: This author was responsible for conception and design and revising the manuscript for important intellectual content.

This manuscript was handled by: Avery Tung, MD.

\section{ACKNOWLEDGMENTS}

The authors thank Prof. Nian-Fang Lu for providing data from the study of Lu et al and Prof. Xiao-Zhi Wang for providing data from the study of Wang et al.

\section{REFERENCES}

1. Angus DC, van der Poll T. Severe sepsis and septic shock. N Engl J Med 2013;369:840-51

2. Kaukonen KM, Bailey M, Suzuki S, Pilcher D, Bellomo R. Mortality related to severe sepsis and septic shock among critically ill patients in Australia and New Zealand, 2000-2012. JAMA 2014;311:1308-16

3. Levy MM, Rhodes A, Phillips GS, Townsend SR, Schorr CA, Beale R, Osborn T, Lemeshow S, Chiche JD, Artigas A, Dellinger RP. Surviving Sepsis Campaign: association between performance metrics and outcomes in a 7.5-year study. Intensive Care Med 2014;40:1623-33

4. Barochia AV, Cui X, Vitberg D, Suffredini AF, O'Grady NP, Banks SM, Minneci P, Kern SJ, Danner RL, Natanson C, Eichacker PQ. Bundled care for septic shock: an analysis of clinical trials. Crit Care Med 2010;38:668-78

5. Dellinger RP, Levy MM, Rhodes A, Annane D, Gerlach H, Opal SM, Sevransky JE, Sprung CL, Douglas IS, Jaeschke R, Osborn TM, Nunnally ME, Townsend SR, Reinhart K, Kleinpell RM, Angus DC, Deutschman CS, Machado FR, Rubenfeld GD, Webb SA, Beale RJ, Vincent JL, Moreno R; Surviving Sepsis Campaign Guidelines Committee including the Pediatric Subgroup. Surviving sepsis campaign: international guidelines for management of severe sepsis and septic shock: 2012. Crit Care Med 2013;41:580-637

6. Rivers E, Nguyen B, Havstad S, Ressler J, Muzzin A, Knoblich B, Peterson E, Tomlanovich M; Early Goal-Directed Therapy Collaborative Group. Early goal-directed therapy in the treatment of severe sepsis and septic shock. N Engl J Med 2001;345:1368-77

7. Kortgen A, Niederprüm P, Bauer M. Implementation of an evidence-based "standard operating procedure" and outcome in septic shock. Crit Care Med 2006;34:943-9

8. Jones AE, Focht A, Horton JM, Kline JA. Prospective external validation of the clinical effectiveness of an emergency department-based early goal-directed therapy protocol for severe sepsis and septic shock. Chest 2007;132:425-32

9. Levy MM, Dellinger RP, Townsend SR, Linde-Zwirble WT, Marshall JC, Bion J, Schorr C, Artigas A, Ramsay G, Beale R, Parker MM, Gerlach H, Reinhart K, Silva E, Harvey M, Regan S, Angus DC; Surviving Sepsis Campaign. The Surviving Sepsis Campaign: results of an international guideline-based performance improvement program targeting severe sepsis. Crit Care Med 2010;38:367-74

10. Yealy DM, Kellum JA, Huang DT, Barnato AE, Weissfeld LA, Pike F, Terndrup T, Wang HE, Hou PC, LoVecchio F, Filbin MR,
Shapiro NI, Augus DC. A randomized trial of protocol-based care for early septic shock. N Engl J Med 2014;370:1683-93

11. Peake SL, Delaney A, Bailey M, Bellomo R, Cameron PA, Cooper DJ, Higgins AM, Holdgate A, howe BD, Webb SA, Williams P. Goal-directed resuscitation for patients with early septic shock. N Engl J Med 2014;371:1496-506

12. Mouncey PR, Osborn TM, Power GS, Harrison DA, Sadique MZ, Grieve RD, Jahan R, Harvey SE, Bell D, Bion JF, Coats TJ, Singer M, Young JD, Rowan KM. Trial of early, goal-directed resuscitation for septic shock. N Engl J Med 2015;372:1301-11

13. Jadad AR, Moore RA, Carroll D, Jenkinson C, Reynolds DJ, Gavaghan DJ, McQuay HJ. Assessing the quality of reports of randomized clinical trials: is blinding necessary? Control Clin Trials 1996;17:1-12

14. Early Goal-Directed Therapy Collaborative Group of Zhejiang Province. The effect of early goal-directed therapy on treatment of critical patients with severe sepsis/septic shock: a multi-center, prospective, randomized, controlled study. Zhongguo Wei Zhong Bing Ji Jiu Yi Xue 2010;22:331-4

15. Wang $X Z$, Lü CJ, Gao FQ, Li XH, Yan WF, Ning FY. [Efficacy of goal-directed therapy in the treatment of septic shock]. Zhongguo Wei Zhong Bing Ji Jiu Yi Xue 2006;18:661-4

16. Jones AE, Shapiro NI, Trzeciak S, Arnold RC, Claremont HA, Kline JA; Emergency Medicine Shock Research Network (EMShockNet) Investigators. Lactate clearance vs central venous oxygen saturation as goals of early sepsis therapy: a randomized clinical trial. JAMA 2010;303:739-46

17. Lu N, Zheng R, Lin H, Shao J, Yu J. [Clinical studies of surviving sepsis bundles according to PiCCO on septic shock patients]. Zhonghua Wei Zhong Bing Ji Jiu Yi Xue 2014;26:23-7

18. Chen ZQ, Jin YH, Chen H, Fu WJ, Yang H, Wang RT. [Early goal-directed therapy lowers the incidence, severity and mortality of multiple organ dysfunction syndrome]. Nan Fang Yi Ke Da Xue Xue Bao 2007;27:1892-5

19. Higgins JP, Thompson SG. Quantifying heterogeneity in a meta-analysis. Stat Med 2002;21:1539-58

20. Higgins JP, Thompson SG, Deeks JJ, Altman DG. Measuring inconsistency in meta-analyses. BMJ 2003;327:557-60

21. DerSimonian R, Laird N. Meta-analysis in clinical trials. Control Clin Trials 1986;7:177-88

22. Gu WJ, Wang F, Bakker J, Tang L, Liu JC. The effect of goaldirected therapy on mortality in patients with sepsis-earlier is better: a meta-analysis of randomized controlled trials. Crit Care 2014;18:570

23. Zhang L, Zhu G, Han L, Fu P. Early goal-directed therapy in the management of severe sepsis or septic shock in adults: a meta-analysis of randomized controlled trials. BMC Med 2015;13:71

24. Angus DC, Barnato AE, Bell D, Bellomo R, Chong CR, Coats TJ, Davies A, Delaney A, Harrison DA, Holdgate A, Howe B, Huang DT, Iwashyna T, Kellum JA, Peake SL, Pike F, Reade MC, Rowan KM, Singer M, Webb SA, Weissfeld LA, Yealy DM, Young JD. A systematic review and meta-analysis of early goal-directed therapy for septic shock: the ARISE, ProCESS and ProMISe Investigators. Intensive Care Med 2015;41:1549-60

25. Rusconi AM, Bossi I, Lampard JG, Szava-Kovats M, Bellone A, Lang E. Early goal-directed therapy vs usual care in the treatment of severe sepsis and septic shock: a systematic review and meta-analysis. Intern Emerg Med 2015;10:731-43

26. Trzeciak S. Protocol-based care for early septic shock. N Engl J Med 2014;371:385

27. Lilly CM. The ProCESS trial-a new era of sepsis management. N Engl J Med 2014;370:1750-1

28. King KM, Adams BD. ACP Journal Club. In septic shock, early goal-directed or standard protocol-based therapy did not reduce mortality. Ann Intern Med 2014;160:JC9

29. Kayyali A. The use of protocols in septic shock. Am J Nurs 2014;114:69

30. Waechter J, Kumar A, Lapinsky SE, Marshall J, Dodek P, Arabi Y, Parrillo JE, Dellinger RP, Garland A. Interaction between fluids and vasoactive agents on mortality in septic shock: a multicenter, observational study. Crit Care Med 2014;42:2158-68 
31. Jansen TC, van Bommel J, Schoonderbeek FJ, Sleeswijk Visser SJ, van der Klooster JM, Lima AP, Willemsen SP, Bakker J; LACTATE Study Group. Early lactate-guided therapy in intensive care unit patients: a multicenter, open-label, randomized controlled trial. Am J Respir Crit Care Med 2010;182:752-61
32. Pope JV, Jones AE, Gaieski DF, Arnold RC, Trzeciak S, Shapiro NI; Emergency Medicine Shock Research Network (EMShockNet) Investigators. Multicenter study of central venous oxygen saturation $(\mathrm{ScvO}(2))$ as a predictor of mortality in patients with sepsis. Ann Emerg Med 2010;55: 40-46.e1 